\title{
Impact De La Motivation Financière Du Personnel De Santé Sur La Qualité Des Soins Administrés Aux Patients A L'hôpital Général De Référence De Kalemie (RDC)
}

\section{[Impact of the Financial Motivation of Health Personnel on the Quality of Care Administered to Patients at Kalemie General Referral Hospital (DR of Congo)]}

\author{
Héman KABEMBA BUKASA ${ }^{1}$, Gaston ALIMASI YUMA ${ }^{2 \dagger}$, David YAMUNYINGA KABINGIE ${ }^{3}$, \\ KILIMA KUNDA Sylvain ${ }^{4}$, KITENGIE NSOMBWE LUTHER ${ }^{1}$, EBONDO EBONDO Gentil ${ }^{5}$, \\ MUTAMBA EYAMBA Christophe ${ }^{6}$ \\ ${ }^{1}$ Section des Sciences Infirmières, Institut Supérieur des Techniques Médicales de Lubao, Lubao, République \\ Démocratique Congo \\ ${ }^{2}$ Faculté de Médecine, Université de Kalemie, Kalemie, République Démocratique Congo : Paix à son âme \\ ${ }^{3}$ Section de Santé communautaire, Institut Supérieur des Techniques Médicales de Kalemie, Kalemie, \\ République Démocratique Congo \\ ${ }^{4}$ Section de Gestion des Institutions de Santé, Institut Supérieur des Techniques Médicales de Kalemie, Kalemie, \\ République Démocratique Congo \\ ${ }^{5}$ Section d'Enseignement et Administration en Sciences Infirmières, Institut Supérieur des Techniques Médicales \\ de Lubao, Lubao, République Démocratique Congo \\ ${ }^{6}$ Section d'Enseignement et Administration en Sciences Infirmières, Institut Supérieur des Techniques Médicales \\ de Kalemie, Kalemie, République Démocratique Congo
}

Résumé

Introduction : La motivation (financière et/ou non financière) demeure l'élément essentiel qui permette au personnel de santé de réaliser son travail avec objectivité, éthique et respect strict des règles déontologiques. Cette étude avait pour but de contribuer à l'amélioration des prestations de soins par les personnels de santé.

Méthodes : Il s'agissait d'une étude descriptive et analytique de cohorte prospective réalisée parmi les personnels de santé de l'hôpital général de référence de Kalemie (RDC) d'Octobre 2016 à Juin 2017, soit huit mois.

Résultats : Sur 102 personnels de santé interrogés, 49 (soit 48\%) sont satisfaits de leur situation financière. Seulement 21 personnels de santé (soit 20,6\%) perçoivent la rémunération complète faite des primes de risque et salaire en provenance du niveau national. L'écart important de prime de risque entre les infirmiers et médecins demeure un obstacle à l'harmonie réelle dans la prestation des services.

Les salaires ne permettent pas aux personnels interrogés de satisfaire les besoins mensuels et on assiste à des frustrations et violation des règles éthiques et déontologiques. Dans la majorité des cas, les soins aux malades étaient continus et intègres, les heures de soins respectées, et la régularité au service respectée. Cette performance se justifie par l'expérience professionnelle, le dévouement aux malades et les attentes de l'amélioration des conditions de vie et de travail des personnels de santé. Par contre les soins n'étaient pas globaux et l'accueil des malades était de mauvaise qualité.

Conclusion : Les résultats de cette étude témoignent d'un besoin urgent d'organisation et d'amélioration des conditions de travail des personnels de santé, ce qui va les permettre de réaliser les activités de soins aux 
malades avec professionnalisme, éthique et respect des règles déontologiques. L'impact considérable consiste à éviter les mouvements incontrôlés des grèves.

Mots-clés : Motivation; Soins Administrés; Personnel de santé ; Kalemie; République Démocratique du Congo.

\begin{abstract}
Introduction: Motivation (financial and / or non-financial) remains the essential element that allows health personnel to carry out their work with objectivity, ethics and strict respect of profession rules. The aim of this study was to help improve the provision of care by health workers.

Methods: This was a prospective cohort descriptive and analytical study carried out among health personnel at the Kalemie General Referral Hospital (DRC) from October 2016 to June 2017, i.e. eight months.

Results: Out of 102 health personnel questioned, 49 (or 48\%) are satisfied with their financial situation. Only 21 health personnel $(20.6 \%)$ receive full compensation made up of risk premiums and salary from the national level. The large gap in risk premium between nurses and doctors remains an obstacle to real harmony in service delivery. Salaries do not allow the staff questioned to meet monthly needs and there is frustration and violation of ethical and deontological rules. In the majority of cases, patient care was continuous and honest, hours of care respected, and regularity of service respected. This performance is justified by professional experience, dedication to patients and the expectations of improving the living and working conditions of health personnel. On the other hand, the care was not comprehensive and the reception of patients was of poor quality.

Conclusion: The results of this study show an urgent need to organize and improve the working conditions of health personnel, which will enable them to carry out patient care activities with professionalism, ethics and respect of deontological rules. The considerable impact is to avoid the uncontrolled movements of strikes.
\end{abstract}

Keywords: Motivation; Care Administered; Personal Health; Kalemie; Democratic Republic of Congo.

\title{
1. INTRODUCTION
}

L'idée de la motivation est très ancienne mais la nature de sa compréhension évolue avec le temps. Platon l'avait évoqué dans sa division tripartite de l'âme [1]. Les théories des besoins et de motivation (Abraham Maslow, Douglas McGrejor, Frederick Herzberg, David MCclelland, Vroom, Adams, Burrhus Skinner, etc.) se focalisent sur les principes sociologiques de l'influence que joue les besoins sur les motivations de l'homme face à son travail [2][3].

Pour Emmanuel KANT, la motivation a deux origines que sont le devoir et la satisfaction du désir, appelée motivation sensible[4].Pour tout travailleur, peu importe le domaine d'activité, il a été établit que la rémunération ( facteur de motivation classique, indiscutable, durable et levier des performances), les conditions de travail, l'implication dans le processus de planification, la culture sociale, le type de projet ou travail, le management du chef hiérarchique, la communication (vision claire et dialogue sociale), la promotion en grade et le développement des compétences professionnelles constituent les sources de la motivation [2][3][5].

Par contre, le manque de reconnaissance, les conditions inappropriées de travail, la suppression de prime, des objections aux propositions de l'agent, le manque de communication et l'insuffisance de rémunération par rapport au travail exécuté constituent la source de démotivation et parfois de recours des agents en grève professionnelle [2][5]6].

Il existe une corrélation entre le degré de motivation et la qualité des prestations des travailleurs. Cet à cet effet qu'on évoque que l'attitude et les orientations du travailleur (en particulier le personnel de santé en ce qui concerne cette étude) déterminent la productivité de l'entreprise, afin d'atteindre les objectifs fixés par avance.

En santé humaine, de par le monde où l'espèce Homo sapiens habite, la motivation est vitale et concerne tous les intervenants de soins en englobant aussi le patient.

Le milieu hospitalier est plein des contraintes à la fois physiques, psychologiques et sociales entre la satisfaction du malade et celle du personnel de santé. A ce point de vue, la démotivation peut être la source de violation des règles de déontologie et d'Ethique parmi les prestataires (infirmiers, médecins, pharmaciens) d'une part, et les obligations d'un agent public de l'Etat [7]-[10].

En République Démocratique du Congo, les études qui abordent la thématique de la motivation des personnels de santé demeurent encore moins nombreuses. Pour Karemere en 2015 [11], les qualités humaines (honnêteté, 
compétence, comportement relationnel, discipline, motivation) et la performance du personnel de santé sont essentielles pour les soins de qualité.

Nous avons mené cette étude enfin d'évaluer l'impact de la motivation financière du personnel de santé de Kalemie sur la finalité de soins offerts aux malades. Les résultats vont permettre aux décideurs d'avoir des informations pertinentes devant guider leurs décisions dans la gestion des ressources humaines.

\section{METHODES}

\subsection{Cadre de l'étude}

Cette étude a été menée à l'Hôpital Général de Reference de Kalemie, dans la Province du Tanganyika, au SudEst de la République Démocratique du Congo. Il s'agissait d'une structure Hospitalière ayant le paquet complémentaire d'activités (PCA), accueillant les malades du Territoire et de ville de Kalemie.

L'Hôpital général de Référence de Kalemie a une capacité d'accueil de 390 lits montés.

\subsection{Type Et Période d'étude}

Il s'agissait d'une étude descriptive et analytique de cohorte prospective. Pour la collecte des données, l'entretien direct ou dirigé et la technique d'échantillonnage stratifié avaient été retenues, soit 6 strates qui représentent les services hospitaliers ci-après : Pédiatrie (26 agents), Médecine interne (18 agents), Chirurgie (21 agents), Urgences (6 agents), Clinique Aisée (15) et Gynécologie et obstétrique (15 agents).

La période d'étude a été de huit mois, soit d'Octobre 2016 à Juin 2017.

\subsection{Population et variables d'étude}

Notre population d'étude a été constituée des personnels de santé de l'hôpital général de référence de Kalemie. D'après nos strates, 102 agents ont été interrogés et constituent notre échantillon. Ils devraient remplir un questionnaire. Les variables d'étude ci-après ont été retenues :

- Facteurs sociodémographiques : Age Sexe, Etat matrimonial, Ancienneté dans la profession, poste occupé au moment de l'enquête, Etudes faites.

- Facteurs liés au traitement socioprofessionnel : Possession du Numéro Matricule de la fonction publique, rémunération, Dépenses moyennes mensuelles, satisfaction de la rémunération financière par rapport au travail exercé.

- Facteurs liés à la qualité de soins : Globalité des soins, continuité de soins, respect de l'ordinogramme de soins protocole du ministère de la santé publique.

Dans la récolte des données, seuls les médecins, les infirmiers, les matrones, les techniciens de radiologie de laboratoire d'analyses biomédicales, les Nutritionnistes, les réceptionnistes et les préposés (dépositaires) à la pharmacie engagée à l'HGR de Kalemie avaient été retenus et inclus. Les médecins et infirmiers stagiaires, les administrateurs gestionnaires et les hygiénistes ont été exclus de cette étude.

Le personnel de santé non satisfait niveau de satisfaction faible par la rémunération perçue devaient constituer la population exposée à offrir les soins de mauvaise qualité aux patient, tandis que ceux qui se disaient satisfait par la rémunération (niveau de satisfaction élevé) parce que ont été considéré comme la population non exposé à offrir les soins de santé de mauvaise qualité. La qualité de soins a été jugée bonne (si les soins offerts aux patients étaient efficaces, intégrés, continus au globaux au mauvaise.

Avant d'entamer l'étude proprement-dite, nous avions effectué une enquête ilote sur un groupe de dix personnels de santé (qui a été exclu de l'étude). Ce prétestavait permis de corriger quelques erreurs par rapport à notre objectif et d'adapter le questionnaire.

\subsection{Analyse statistique des données}

Les données récoltées dans le questionnaire ont été encodés dans un fichier Excel (Microsoft 2016, USA). Nous avons fait usage de calcul de la moyenne et P-value au seuil de 0,05. L'Epi-Info version 7.2 a été utilisé. 


\subsection{Définitions opérationnelles}

- Soins continus : Soins administrés aux personnes malades (souffrantes) ou à risque de souffrir du moment de contact jusqu'à la fin de l'épisode de maladie ou de risque.

- Soins globaux : soins administrés aux patients en prenant en compte l'aspect biologique (biomédical) et psychosocial.

- Motivation financière : la somme représentative de l'ensemble des gains susceptibles d'être évalués en espèce (Prime National de l'Etat/ gouvernement + Prime locale des entrées financières+ Prime de risque et fixés par accord au par des dispositions légales et règlementaires qui sont dus en vertu d'un contrat de travail entre l'employeur et l'employé qui pour ce dernier à assumer ses responsabilités positivement ou négativement.

- Personnel de santé : c'est l'ensemble des personnes opérationnelles (médecins, infirmiers, Matrones, accoucheuses traditionnelles, techniciens de laboratoire, nutritionnistes, Kinésithérapeutes, Réceptionnistes préposé à la pharmacie engagé à l'HGR de Kalemie. Les hygiénistes, les administrateurs, gestionnaires et les autres catégories administratives n'ont pas été retenus.

\subsection{Ethique}

L'accord des autorités du Bureau Central de la Zone de Santé (BCZS) de Kalemie et de l'HGR de Kalemie avait été obtenu. Le consentement éclairé des enquêtés étaient obtenus, l'anonymat et la confidentialité des réponses avaient été garantis.

\section{RESULTATS}

Les résultats de cette étude renseignent que sur 102 personnels de santé interrogés, $48 \%$ (soit 49 personnes) seulement étaient satisfaits de leur situation financière. Les agents bénéficiant des Numéros matricules de la fonction publique représentaient $45,1 \%$ (soit 46 personnes) (Figure 1).

Tableau I. Caractéristiques sociodémographiques et professionnelles

\begin{tabular}{|c|c|c|}
\hline Caractéristiques & $\mathbf{n}$ & $\%$ \\
\hline \multicolumn{3}{|l|}{ Sexe } \\
\hline Masculin & 44 & 43,1 \\
\hline Féminin & 58 & 56,9 \\
\hline \multicolumn{3}{|l|}{ Etat matrimonial } \\
\hline Marié (es) & 95 & 93,2 \\
\hline Célibataires & 5 & 4,9 \\
\hline Veufs (ves) & 2 & 2,0 \\
\hline \multicolumn{3}{|l|}{ Profession : Poste occupé } \\
\hline Médecin & 20 & 19,6 \\
\hline Infirmier (e) & 71 & 69,6 \\
\hline Technicien radiologue & 3 & 2,9 \\
\hline Réceptionniste & 2 & 2,0 \\
\hline Laborantin & 4 & 3,9 \\
\hline Préposé à la pharmacie & 2 & 2,0 \\
\hline \multicolumn{3}{|l|}{ Expérience professionnelle } \\
\hline$\leq 4$ ans & 12 & 11,8 \\
\hline $5-10$ ans & 24 & 23,5 \\
\hline $11-16$ ans & 45 & 44,1 \\
\hline$\geq 17$ ans & 21 & 20,6 \\
\hline \multicolumn{3}{|l|}{ Etudes faites * } \\
\hline Médecinegénérale & 20 & 19,6 \\
\hline Santé publique AO & 1 & 1,0 \\
\hline Santé publique A 1 & 1 & 1,0 \\
\hline Techniques de laboratoire A1 & 1 & 1,0 \\
\hline Infirmières $\mathrm{A} 0$ & 1 & 1,0 \\
\hline Infirmières A1 & 29 & 28,4 \\
\hline Infirmières A2 & 23 & 22,5 \\
\hline Infirmières A3 & 25 & 24,5 \\
\hline Humanités / D6 & 1 & 1,0 \\
\hline
\end{tabular}


*AO =Licence ou Baccalauréat +5 des Etudes supérieures et universitaires ; Al= Graduat ou Baccalauréat +3 des Etudes supérieures et universitaires ; A2=Diplômes d'Etat des humanités techniques médicales ; A3 = Certificat des humanités techniques médicales ; D6= Diplômes d'Etat des humanités Non médicales.

La majorité des agents $45,1 \%$ (soit 46 personnes) percevaient la prime de risque et la prime locale durant la période d'étude, 21 personnels de santé seulement (20,6\%) avaient reconnus avoir l'habitude de percevoir la rémunération complète faite du salaire de l'état, le prime de risque professionnel et prime local (de l'HGR de Kalemie selon les entrées mensuelles).

Tableau II. Rémunération et dépenses moyennes

\begin{tabular}{|c|c|c|}
\hline Rémunération et dépenses & $\mathbf{n}$ & $\%$ \\
\hline \multicolumn{3}{|c|}{ Types de Rémunération mensuelle * } \\
\hline $\mathrm{SE}+\mathrm{PR}+\mathrm{PL}$ & 21 & 20,6 \\
\hline $\mathrm{PR}+\mathrm{PL}$ & 46 & 45,1 \\
\hline PL & 35 & 34,3 \\
\hline \multicolumn{3}{|c|}{ Montant de rémunération mensuelle (USD) } \\
\hline $30-58,8$ & 65 & 63,7 \\
\hline $59-88,2$ & 11 & 10,8 \\
\hline $88,8-117,6$ & 6 & 5,9 \\
\hline$\geq 118$ & 20 & 19,6 \\
\hline \multicolumn{3}{|c|}{ Dépenses familiales moyennes (USD) } \\
\hline $30-58,8$ & 11 & 10,8 \\
\hline $59-88,2$ & 32 & 31,4 \\
\hline $88,8-117,6$ & 37 & 36,3 \\
\hline$\geq 118$ & 22 & 21,6 \\
\hline
\end{tabular}

* SE= salaire de l'Etat $; P R=$ Prime de risque professionnel ; $P L=$ Prime locale de l'hôpital

$* * U S D=$ United state dollars, payés en francs congolais $(C D F)$ au taux de 1700 CDF

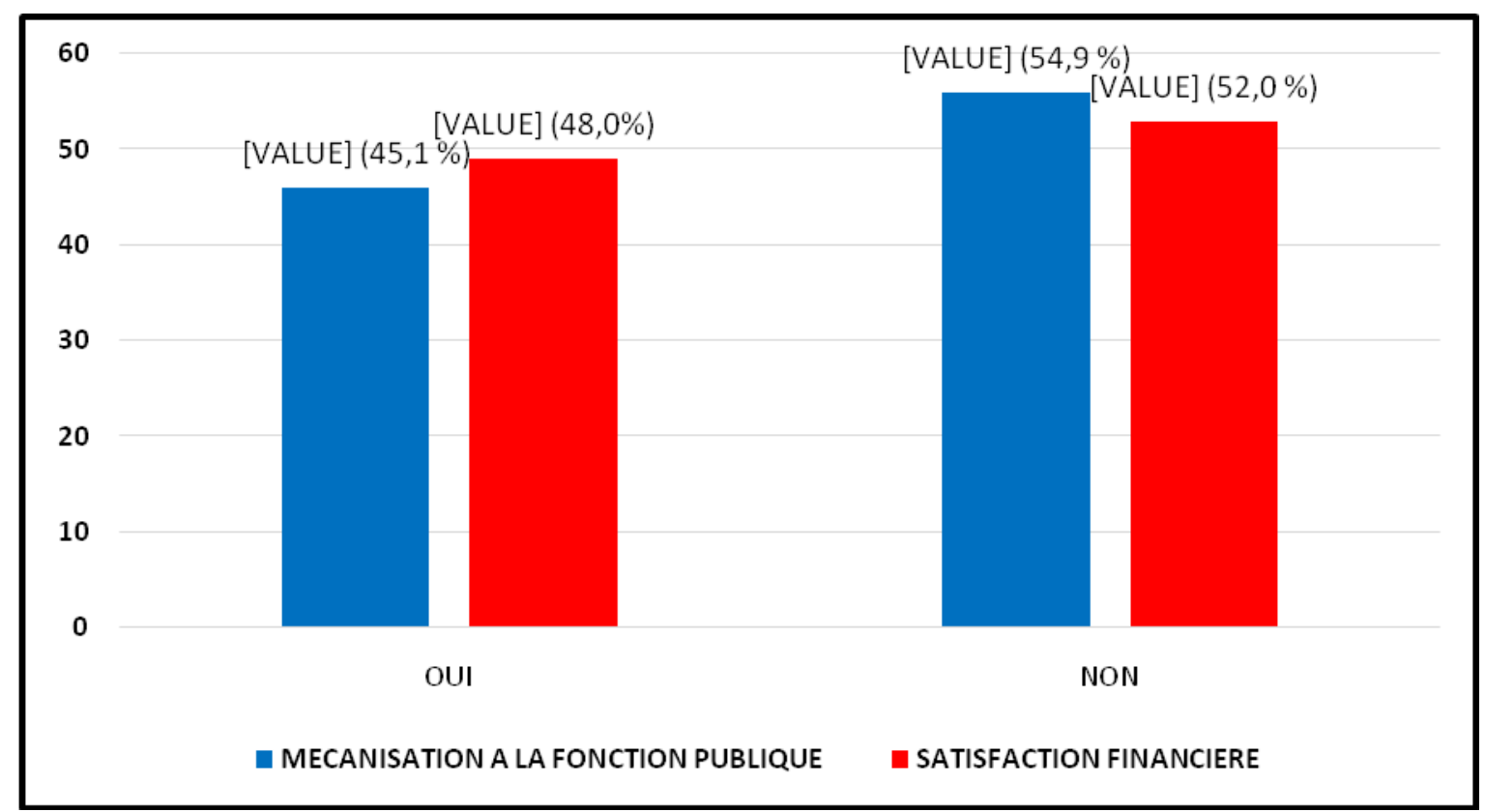

Figure 1. Mécanisation à la fonction publique et satisfaction du personnel de santé

Les résultats de cette étude révèlent que 65 personnels de santé $(63,7 \%)$ ont de rémunération mensuelle comprise entre 30 à 58,8 USD, alors que les dépenses familiales représentent en majorité 59 à 88,2 USD dans une proportion de 36,3\% (soit 37 personnels de santé). Il existe néanmoins 20personnels de santé dont la rémunération mensuelle dépasse 110 USD (19,6\%). Pour combler le déficit entre la rémunération et les besoins de famille, les personnels de santé insatisfaits recouvrent à certaines manœuvres dont la combinaison avec un autre travail (ailleurs) dans 20,6\% (21 cas), rançonner le malade en ayant recours à certaines pratiques dans $14,7 \%$ (15 cas et soigner les malades à domicile pour 7,8\% (8 cas). (Tableau II) 
Le profil des enquêtés nous renseigne qu'il s'agissait des personnels de santé dont l'âge variait de 28 à 81 ans avec une moyenne de 45,1 ans, à prédominance féminine $(56,9 \%, 58$ cas sur 102), mariés $(93,2 \%, 95$ cas), infirmiers (e) de profession $(69,6 \%, 71$ cas), avec expérience professionnelle de 11 à 16 ans $(44,1 \%, 45$ cas) et dont les principales études faites étaient des sciences infirmières $(76,5 \% ; 78$ cas) et la médecine générale $(19,6 \%$; 20 cas). (Tableau I)

Les soins des malades ont été continue des 77,56 (79 cas). Pour 71 cas $(69,6 \%)$, les soins ont été intègres avec une différence significative (p 0,05). Les heures de soins ont été respectées dans 76,5\% (78 cas).

Les personnels de santé étaient réguliers dans 64,7\% (66 cas). Les normes établies dans l'ordinogramme ont été respectés dans la proportion de $65,7 \%$ (67 cas).

Par contre, les soins n'étaient globaux (57, 8\%, soit 59 cas). L'accueil des malades n'était pas apprécié et pour 63 cas, soit $61,8 \%$, les patients n'étaient pas bien accueillis. (Tableau III)

Tableau III. Satisfaction du personnel de santé et aux soins des malades

\begin{tabular}{|c|c|c|c|c|c|c|}
\hline \multirow[t]{2}{*}{ Soins } & & \multicolumn{2}{|c|}{ Satisfaction } & \multirow{2}{*}{$\begin{array}{r}\text { Total } \\
\mathrm{n}(\%)\end{array}$} & \multirow[t]{2}{*}{$\mathrm{IC}_{95}$} & \multirow[t]{2}{*}{ p-value } \\
\hline & & $\begin{array}{c}\text { OUI } \\
\mathrm{n}(\%)\end{array}$ & $\begin{array}{l}\text { NON } \\
\mathrm{n}(\%)\end{array}$ & & & \\
\hline \multirow[t]{2}{*}{ Régularité du personnel au travail } & OUI & $34(69,4)$ & $\begin{array}{c}32 \\
(60,4)\end{array}$ & $\begin{array}{c}66 \\
(64,7)\end{array}$ & $\begin{array}{c}1,49 \\
{[0,65-3,338]}\end{array}$ & $\mathrm{O}, 457$ \\
\hline & NON & $\begin{array}{c}15 \\
(30,6)\end{array}$ & $\begin{array}{c}21 \\
(39,6)\end{array}$ & $\begin{array}{c}36 \\
(35,3)\end{array}$ & & \\
\hline \multirow[t]{2}{*}{ Continuité des soins } & OUI & $\begin{array}{c}29 \\
(59,2)\end{array}$ & $\begin{array}{c}50 \\
(94,3)\end{array}$ & $\begin{array}{c}79 \\
(77,5)\end{array}$ & $\begin{array}{c}0,08 \\
{[0,02-0,32]}\end{array}$ & 0,0000 \\
\hline & NON & $\begin{array}{c}20 \\
(40,8)\end{array}$ & $\begin{array}{c}3 \\
(5,7)\end{array}$ & $\begin{array}{c}23 \\
(22,5)\end{array}$ & & \\
\hline \multirow[t]{2}{*}{ Globalité des soins } & OUI & $\begin{array}{c}26 \\
(56,1)\end{array}$ & $\begin{array}{c}17 \\
(32,1)\end{array}$ & $\begin{array}{c}43 \\
(42,2)\end{array}$ & $\begin{array}{c}2,39 \\
{[1,07-5,35]}\end{array}$ & 0,05 \\
\hline & NON & $\begin{array}{c}23 \\
(40,9)\end{array}$ & $\begin{array}{c}36 \\
(67,9)\end{array}$ & $\begin{array}{c}59 \\
(57,8)\end{array}$ & & \\
\hline \multirow[t]{2}{*}{ Respect de l'ordinogramme } & OUI & $\begin{array}{c}34 \\
(69,4)\end{array}$ & $\begin{array}{c}33 \\
(62,3)\end{array}$ & $\begin{array}{c}67 \\
(65,7)\end{array}$ & $\begin{array}{c}1,37 \\
{[0,60-3,12]}\end{array}$ & 0,583 \\
\hline & NON & $\begin{array}{c}15 \\
(30,6)\end{array}$ & $\begin{array}{c}20 \\
(37,7)\end{array}$ & $\begin{array}{c}35 \\
(34,3)\end{array}$ & & \\
\hline \multirow[t]{2}{*}{ Intégrité des soins (intègres) } & OUI & $\begin{array}{c}40 \\
(81,6)\end{array}$ & $\begin{array}{c}31 \\
(58,5)\end{array}$ & $\begin{array}{c}71 \\
(69,6)\end{array}$ & $\begin{array}{c}3.15 \\
{[1,27-7,81]}\end{array}$ & 0,020 \\
\hline & NON & $\begin{array}{c}9 \\
(18,4)\end{array}$ & $\begin{array}{c}22 \\
(41,5)\end{array}$ & $\begin{array}{c}31 \\
(30,4)\end{array}$ & & \\
\hline \multirow[t]{2}{*}{ Bon Accueil des patients } & OUI & $\begin{array}{c}19 \\
(38,8)\end{array}$ & $\begin{array}{c}20 \\
(37,7)\end{array}$ & $\begin{array}{c}39 \\
(38,2)\end{array}$ & $\begin{array}{c}1,04 \\
{[0,47-2,32]}\end{array}$ & 1,000 \\
\hline & NON & $\begin{array}{c}30 \\
(61,2)\end{array}$ & $\begin{array}{c}33 \\
(62,3)\end{array}$ & $\begin{array}{c}63 \\
(61,8)\end{array}$ & & \\
\hline \multirow[t]{2}{*}{ Respect de l'heure de soins } & OUI & $\begin{array}{c}40 \\
(81,6)\end{array}$ & $\begin{array}{c}38 \\
(71,7)\end{array}$ & $\begin{array}{c}78 \\
(76,5)\end{array}$ & $\begin{array}{c}1,75 \\
{[0,69-4,48]}\end{array}$ & 0,343 \\
\hline & NON & $\begin{array}{c}9 \\
(18,4)\end{array}$ & $\begin{array}{c}15 \\
(28,3)\end{array}$ & $\begin{array}{c}24 \\
(23,5)\end{array}$ & & \\
\hline
\end{tabular}




\section{DISCUSSION}

Dans tous les domaines de la vie, la motivation demeure indissociable du rendement[12]-[14]. Le personnel de santé motivé constitue une réussite potentielle dans les soins apportés aux malades. En outre, la motivation est facteur de fidélisation du personnel et d'accroissement des performances de la structure des soins [2][13][15]. La motivation est tributaire des facteurs financiers et non financiers [12][16][17], ce qui implique le management du gestionnaire des ressources humaines[2]. Toutefois, nombreuses études démontrent que, de nos jours, la motivation financière occupe une place de choix [6][7][15].

La première étape pour bénéficier le salaire de l'Etat est d'avoir le numéro matricule, une fierté d'être reconnu par son employé. A l'hôpital général de référence de Kalemie, la proportion des agents matriculés (sous statut) est moindre, soit $45,1 \%$. Cette situation constitue un handicap non négligeable à la motivation des agents de santé, et par conséquent de l'atteinte des objectifs du millénaire pour le développement [7][18][19].

Cette étude révèle la très moindre proportion des personnels de santé recevant la rémunération complète (prime de risque professionnel, prime locale institutionnelle et salaire de 1'Etat), soit 20,6\%. Cela devrait interpeller les décideurs car toute démotivation a des impacts non négligeables, allant principalement de la baisse des performances individuelles, la démission secteur public vers le secteur privé, la corruption, et la migration des personnels de santé vers les centres urbains, pénalisant ainsi les milieux ruraux qui sont en majorité peuplés [3][6][7][18].

A l'Hôpital Général de Référence de Kalemie, il existe une nette balance négative entre les revenues et les dépenses moyennes des enquêtés. Les personnels de santé dépensent plus que ce qu'ils gagnent. Les médecins, avec prime de risque, semblent mieux servi car celle-ci demeure au-delà de cinq cents dollars américains. Par contre, les infirmiers et les autre personnels administratifs sont moins rémunérés, surtout ceux qui ne sont pas éligibles à la prime de risque. Ce grand écart constitue donc une source de frustration, démotivation et conflits potentiels entre les médecins et les infirmiers, les personnels administratifs et techniques qui sont par-dessus plus nombreux, comme le souligne Mathauer et Imhoff.[7] : 22\% des médecins contre 78\% des infirmiers au Kenya, et $11 \%$ des médecins contre $89 \%$ des infirmiers au Benin.

Dans une étude Chypriote, Lambrou et al.[20]souligne que le médecin sont moins satisfaits que les infirmiers, ce qui contraste avec la réalité pragmatique congolaise évoquée dans cette étude.

Dans le souci de couvrir les besoins familiaux de base, la majorité des personnels de santé recourent àcertaines pratiques informelles [6][18]dont la combinaison avec un autre métier, soigner les malades à domicile et rançonner les malades hospitalisés en conditionnant les soins par la motivation financières soignant- soigné. Ces manœuvres, peu éthique et déontologique, ne sont pas à encourager. Elles relèvent des faiblesses qui découlent de l'insatisfaction des besoins. C'est par là qu'une nécessité absolue de satisfaire les travailleurs de santé s'impose, afin de favoriser les soins de qualité [3][6][7][11][14].

La qualité de soins est tributaire des ressources humaines de qualité et de l'environnement de travail [7]. Les personnels de santé ont la responsabilité absolue, en tant qu'acteurs incontournables dans l'administration des soins de produire les soins de qualité au bénéfice des malades [14][17].

Les personnels de santé, en majorité des infirmiers et médecins, fournissent malgré les facteurs favorables à la grande démotivation, des efforts non négligeables. Les soins demeurent en majorité continus et intégrés, les heures d'administrations des soins respectées en grande majorité et les personnels encore réguliers au travail. Nous osons croire que les obligations morales vis-à-vis de la communauté, éthiques et déontologiques ont beaucoup contribué. En outre, l'espoir d'un lendemain meilleur n'est pas à écarter[7].

Par contre, les soins étaient légèrement non globaux et les malades mal accueillis. Ces facteurs peuvent être associés à la démotivation du personnel des personnels de santé. Toutefois, soulignons qu'un bon accueil n'est pas uniquement tributaire de la motivation financière. Les facteurs éthiques et déontologiques occupent le premier rang [3][11][21].

Pour Mathauer et Imhoff.[7], " les agents de santé démotivés et frustrés sont incapables de satisfaire les objectifs dévolus ". D'où les conclusions de Dieleman et al.[3]s'avèrent capitales, à savoir les mesures incitatives financières et non financières et l'amélioration de l'environnement de travail. Dans une étude Marocaine, Belrhiti et Chagar [21]préconisent de tenir compte de tous les éléments de ressources humaines pour motiver les travailleurs : la responsabilité du travail par le travailleur (personnel de santé dans notre série d'étude), la relation aux subordonnées, la considération du leader (chef hiérarchique), la possibilité d'avancement en grade, la reconnaissance des efforts fournis par les primes et décoration (mérites), l'autonomisation de la prise de décision et la sécurité du personnel. 


\section{LIMITES DE CETTE ETUDE}

Les limites de cette étude comprennent:(1) Questionnaire non standard; (2) Données quantitatives non complétées par les données qualitatives; (3) Etude ne met pas en corrélation les conditions de travail, la satisfaction et la motivation ; (4) se résume dans l'unique aspect financier.

\section{CONCLUSION}

Pour réaliser adéquatement les objectifs fixés dans l'exercice de la profession parmi les professionnels de santé, la motivation demeure indéniablement l'élément essentiel reconnus dans le mangement des ressources humaines. Les résultats de cette étude sont une interpellation aux autorités Polito administratives et les décideurs en santé car ils révèlent que les personnels de santé sont insatisfaits et démotivés suite aux conditions financières et cela impact négativement la réalisation de la majorité des aspects de soins aux malades. A cet effet, nous recommandons, outre l'amélioration des conditions de vie des personnels de santé, les études internes et externes tendant à identifier les facteurs de motivation et de démotivation dans notre milieu.

\section{REMERCIEMENT}

Nous tenons à remercier Isidore KULANGA TUNDWA pour sa contribution non négligeable dans la récolte des données ayant servis dans l'élaboration de cette étude.

\section{REFERENCES}

[1] J. Cooper, "La théorie Plutonienne de la motivation humaine », Revue Philosophique de France et de l'Etranger 1991, 181 (4) : 517543

[2] H. Peters David, S. Chakraborty , P. Mahapatra , L. Steinhardt,"Job satisfaction and motivation of health workers in public and private sectors: cross-sectional analysis from two Indian states", Human Resources for Health 2010 8:27 https://doi.org/10.1186/1478-4491-827

[3] M. Dieleman, P.-V. Cuong, L.-V. Anh, T. Martineau, "Identifying factors for job motivation of rural health workers in North Viet Nam",Human Resources for Health 2003, 5(1):10.

[4] B. Gnassounou, "La raison, motivation et morale dans la philosophie morale analystique ", In histoire raisonnée de la philosophie morale et politique, Edition La Découverte 2001 : 734-741

[5] Q. Luzurier, C. Damm, F. Lion, C. Daniel, L. Pellerin and M.-P. Tavolacci," Strategy for recruitment and factors associated with motivation and satisfaction in a randomized trial with 210 healthy volunteers without financial compensation"BMC Medical Research Methodology 2015, 15:2 https://doi.org/10.1186/1471-2288-15-2

[6] S. Stringhini, S. Thomas, P. Bidwell, T. Mtui and A. Mwisongo, "Understanding informal payments in health care: motivation of health workers in Tanzania", Human Resources for Health 2009, 7:53 https://doi.org/10.1186/1478-4491-7-53

[7] I. Mathauer and I. Imhoff, "Health worker motivation in Africa: the role of non-financial incentives and human resource management tools", Human Resources for Health 2006, 4:24 https://doi.org/10.1186/1478-4491-4-24

[8] M. Bogren, M. Grahn, B.-B. Kaboru and M. Berg, "Midwives' challenges and factors that motivate them to remain in their workplace in the Democratic Republic of Congo — an interview study ", Human Resources for Health 2020, 18 : 65

[9] W. Laubach, S. Fischbeck, "Job satisfaction and the work situation of physicians: a survey at a German university hospital", International Journal of Public Health 2007, 52: 54-59

[10] A. Paris, J.-L. Bosson, MS. Melis, A. Moreau-Gaudry, M. Hommel, J.-L. Cracowski, "Evolution of the satisfaction of subjects enrolled in clinical studies", Therapie 2006, 61:419-423

[11] H. Karemere, "Analyse des attitudes et comportements des médecins et infirmiers en tant que levier stratégique de la gestion des ressources hospitalières"Pan African Medical Journal, 2015, 21:193 https://doi.org/10.11604/pamj.2015.21.193.6026

[12] A. Greenspan Jesse, A. McMahon Shannon, J. Chebet Joy, Maurus Mpunga, P. Urassa David and J. Winch Peter, "Sources of community health worker motivation: a qualitative study in Morogoro Region, Tanzania" Human Resources for Health 2013, 11:52 https://doi.org/10.1186/1478-4491-11-52

[13] C. Zedini, A. Ben Cheikh, M. Limam, Y. Henrichi, M. Mellouli, et al., "Les facteurs de motivation au travail chez les cadres paramédicaux dans un hôpital Tunisien ", Santé publique 2016, 28 (5) : 613-622 


\section{DOI: $\underline{10.51386 / 25815946 / i j s m s-v 4 i 5 p 113}$}

Volume: 4 Issue: 5

September to October 2021

https://www.ijsmsjournal.org

[14] B. Galland, "Etude de la motivation des acteurs de la santé", Centre International de Recherche et de Développement, Septembre $2006: 4-26$

[15] I. Agyepong, P. Anafi, E. Asiamah, E.-K. Ansah, D.-A. Ashon, C. Narh-Dometey, "Health worker (internal customer) satisfaction and motivation in the public sector in Ghana", International Journal of Health Planning and Management 2004, 19:319-336.

[16] J.-B. Kahindo Mbeva, C. Schirvel, E. Godelet, A. Wodon, D. Porignon, M. Bonami, "Réforme des structures intermédiaires de santé en République démocratique du Congo ", Santé Publique 2014, 26 (6) : 849-858

[17] I. Proper Karin, J.-H. Deeg Dorly and J. Allard van der Beek, "Challenges at work and financial rewards to stimulate longer workforce participation", Human Resources for Health 2009, 7:70 https://doi.org/10.1186/1478-4491-7-70

[18] R. Kaba Alhassan, N. Spieker, P.Van Ostenberg, A. Ogink, E. Nketiah-Amponsah and F Tobias Rinke de Wit,"Association between health worker motivation and healthcare quality efforts in Ghana", Human Resources for Health 2013, 11:37 https://doi.org/10.1186/1478-4491-11-37

[19] I.-R. Chandler, C.-J. Kizito, L. Taaka, C. Nabirye, M. Kayendeke, D. DiLiberto and G. Sarah Staedke, "Aspirations for quality health care in Uganda: How do we get there?", Human Resources for Health 2013, 11:13 https://doi.org/10.1186/1478-4491-11-13

[20] P. Lambrou, N. Kontodimopoulos, D. Niakas, "Motivation and job satisfaction among medical and nursing staff in a Cyprus public general hospital", Human Resources for Health 2010, 8:26 https://doi.org/10.1186/1478-4491-8-26

[21] Z. Belrhiti et H. Chagar, "Les facteurs de motivation des soignants", Gestions hospitalières 2013, 529 : 34-37 